\title{
Digital Image Watermarking Scheme Based on LWT and DCT
}

\author{
Amy Tun and Yadana Thein
}

\begin{abstract}
As a potential solution to defend unauthorized replication of digital multimedia objects, digital watermarking technology is now attracting significant attention. With the aid of a combined Lifting Wavelet Transform (LWT) and Discrete Cosine Transform (DCT), an approach for watermaking scheme to protect copyrights of digital images is presented in this paper. The lifting wavelet transform is applied to decompose the original image into four sub-band images. Then the discrete cosine transform is computed on the selected sub-band of the $L W T$ coefficients. The watermark is embedded in the DCT transformed of the selected LWT sub-band of the cover image. The proposed system focuses on an invisible watermark embedding, imperceptibility of watermarked image and performance evaluation metrics. This presented algorithm is realized in MATLAB.
\end{abstract}

Index Terms-Copyright protection, discrete cosine transform (DCT), frequency-domain watermarking, lifting wavelet transform (LWT)

\section{INTRODUCTION}

Digital watermarking technology for multimedia contents as the field of information hiding technology is becoming a useful way in dealing with the copyright protection problem, due to the rapid growth of such digitized media over the internet.

Digital image watermarking is the process of embedding additional information into an image to make assertion about the image. The embedded information is called watermark which is, in general, a visible or invisible identification code that may contain owner's information. The visible watermark can be easily removed from the digital cover image. Unlike visible watermarks, the invisible watermarks could be very difficult to remove from the media because they became an integral component of the cover image after being embedded. This embedded data can later be extracted from, or detected in, the multimedia for security purposes. A watermarking algorithm consists of the watermark structure, an embedding algorithm, and an extraction or a detection algorithm.

There are two main classes in watermarking techniques, namely the spatial and transform domain. In transform domain, the data is embedded by modulating the transform domain signal coefficients. The spatial domain techniques embed the watermark by directly modifying the pixel values of the original image. The transform domain techniques are most successful and popular for image watermarking. In all transform domain watermarking schemes, there is a conflict between robustness and transparency. If the watermark is

Manuscript received December 30, 2012; revised March 12, 2013.

The authors are with the University of Computer Studies, Yangon, Myanmar (e-mail: amtnyo@gmail.com). embedded in perceptually most significant components, the scheme would be robust to attacks but the watermark may not be meeting imperceptibility criterion. On the other hand, if the watermark is embedded in perceptually insignificant components, it would be easier to hide the watermark but the scheme may be less resilient to attacks. An effective watermarking scheme should meet certain requirements including transparency, robustness, security, and low-computational complexity.

Digital image watermarking schemes can be placed under two categories based on whether or not they use the original image for extraction of watermark from watermarked image such as blind watermarking techniques which do not require original image and non-blind watermarking that requires original image to exist for detection. Two major applications of digital watermarking are copyright protection (proving ownership of data) and data authentication. The quality of watermarked image is measured by Peak Signal to Noise Ratio (PSNR) and Mean Square Error (MSE). The process of watermarking in the simplest form is shown in Fig. 1.

The main objective of this paper is to present a digital image watermarking technique which based on combining two transforms: LWT and DCT. Watermarking is done by altering the wavelets coefficients of carefully selected LWT sub-bands $(\mathrm{HH})$, followed by the application of the DCT transform on the selected sub-bands. The rest of the paper is organized as follows: a brief review of some of the works available in the literature that develop watermarking in frequency domain is presented in section 2. The proposed blind watermarking approach is given in section 3. The experimental results are described in section 4. Finally, the conclusions are shown in section 5 .

\section{RELATED WORK}

A number of earlier works related to digital image watermarking inspired us to do this research. Some of such recent researches are briefly described in this section.

G. Gupta and H. Aggarwal [1] have presented a digital image watermarking based on two-dimensional discrete cosine transform (DCT2), two-dimensional discrete wavelet transform (DWT2) and two dimensional Fast Fourier transform (FFT2). In this system, traces of original and watermarked images have been presented. The proposed algorithm has been observed that two dimensional discrete cosine transform gives the better results than other transforms.

P. Lam, O. Winkelmeyer, S. A. Mehdi and N. kamoosi [2] have described a technique of using perceptual masking in conjuction with DCT. The results of three algorithms based on DCT, DWT and DCT with PM have been analyzed and 
compared. They concluded that the method of DCT with perceptual masking is better than both DCT and DWT.

R. Hovancak and D. Levicky [3] evaluated two different watermarking methods using discrete cosine transformation of image. Original image is needed for extraction of watermark in first method and the one is not needed in second method. The proposed method showed that first method has better robustness but both methods are good candidates for copyright protection.

Y. Jie [4] has proposed an algorithm of image information hiding based on the combination of image blending, discrete cosine transform and new anti-Arnold transform. The proposed system showed that the algorithm has good imperceptibility, validity and certain degree of robustness under some common noise.

S. Hajjara, M. Abdallah and A. Hudaib [5] have presented a method for digital image watermarking using the biorthogonal wavelet transform. The method is based on decomposing an image using the Discrete Wavelet Transform (DWT), and then embedding a watermark into significant coefficients of the transform. The proposed method showed that biorthogonal wavelets have the property of perfect reconstruction and smoothness.

D. Arya [6] has presented a comprehensive survey of the current techniques that have been developed and their effectiveness. Schemes in frequency domain and wavelet domain are introduced with analysis of pros and cons, in terms of imperceptibility, robustness, implementation complexity etc., for each domain. The presented paper showed that these algorithms have a satisfactory performance under image cropping and JPEG lossy compression.

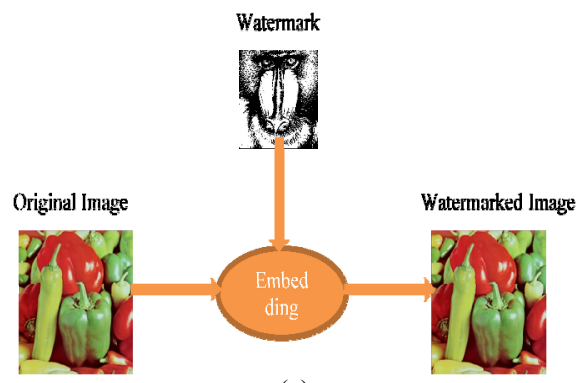

(a)

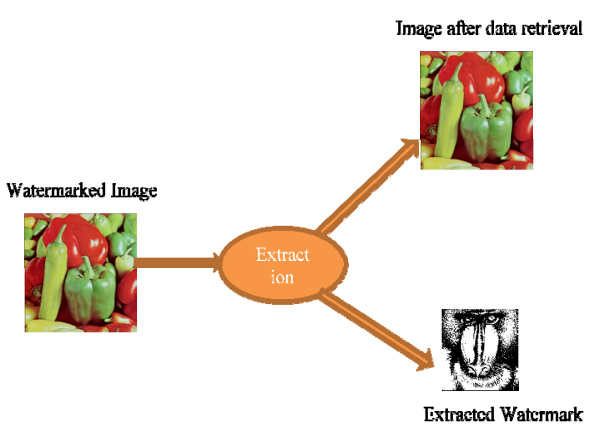

(b)

Fig. 1. Process of watermarking: (a) Embedding and (b) Extraction

The proposed scheme using LWT and DCT works on color images or gray-scale images as the host images, and embeds an image as the watermark. One of the main differences of the proposed system than other watermarking techniques in frequency domain is the host image decomposition by using Lifting Wavelet Transform (LWT). In addition, the proposed scheme chooses the detail coefficients of LWT while other wavelet transform such as DWT watermarking techniques selects the approximation coefficients. Detail coefficient is chosen in order to make the technique robust against attack, and to keep imperceptibility. Moreover, the scheme uses the idea of combining two transform with the aim of improvement of the watermarking performance. The important goal for the system is to preserve imperceptibility and robustness of the watermarked image.

\section{WATERMARKING APPROACH IN FREQUENCY DOMAIN}

This section details the proposed blind watermarking scheme for copyright protection of digital images. The following subsections present the steps involved in the watermark embedding and extraction processes along with a brief description about the Lifting Wavelet Transform (LWT) and Discrete Cosine Transform (DCT).

\section{A. Lifting Wavelet Transform (LWT)}

Lifting Wavelet Transform based on the traditional wavelet is introduced by Wim Sweldens, using a simple relationship among all multi-resolution analyses with the same scaling function. The lifting scheme has several virtues compared with the traditional wavelet such as LWT can compute more effectively and needs smaller memory space and the transform coefficients from LWT are integers, overcoming the weakness of quantizing errors from the traditional wavelet transform [7].

Lifting wavelet transform requires three phases for its implementation, namely: split, predict and update.

Split: The original data set $x[n]$ is divided into two subsets with no common elements, whose length are the half of original data. Generally speaking, the original signal is divided into odd subset $x_{\mathrm{o}}[n]=x[2 n+1]$ and even subset $x_{e}[n]$ $=x[2 n]$.

Predict: Odd series $x_{\mathrm{o}}[n]$ is predicted according to even series $x_{e}[n]$ by the predict operator $P$, and the errors are called wavelet coefficients $d[n]$ as in equation (1).

$$
d[n]=x_{\mathrm{o}}[n]-P\left(x_{e}[n]\right)
$$

Update: Update operators $U$ are put on wavelet coefficients $d[n]$, and then the results add the odd series $x_{e}[n]$, which are called scale coefficients $c[n]$ as in equation (2).

$$
c[n]=x_{e}[n]+U(d[n])
$$

The lifting stage is formed by these three steps. As long as the same $P$ and $U$ are chosen for the forward and inverse transforms, the construction of the original signal will be perfect. Fig. 2 is the process of the lifting wavelet algorithm.

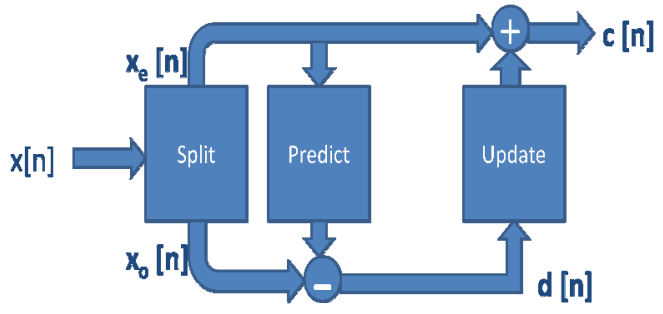

Fig. 2. Lifting wavelet transform 
In lifting wavelet transform, original image decomposition produces four bands of data, such as approximation coefficients matrix (LL) and detail coefficients matrices HL, LH, HH. Host image as shown in Fig. 3 is decomposed into four bands as shown in Fig. 4. Fig. 5 depicts the perfect reconstruction using the approximation coefficients and detail coefficients, obtained by lifting wavelet decomposition.

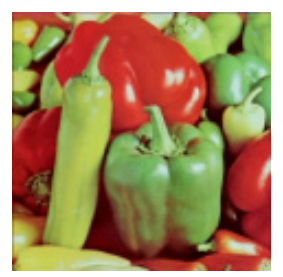

Fig. 3. Original image

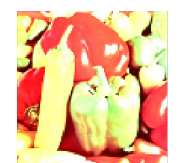

(a)

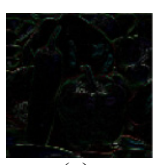

(c)

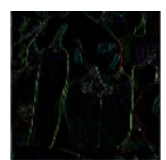

(b)

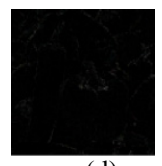

(d)
Fig. 4. (a) Sub-band LL; (b) Sub-band LH; (c) Sub-band HL; (d) Sub-band $\mathrm{HH}$

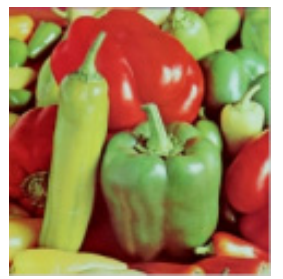

Fig. 5. Original image reconstruction

\section{B. Discrete Cosine Transform (DCT)}

A technique for converting a signal into elementary frequency components is the discrete cosine transform. It represents an image as a sum of sinusoids of varying magnitudes and frequencies. With an input image, $x$, the DCT coefficients for the transformed output image, $y$, are computed according to equation (3) shown below. In the equation, $x$ is the input image having $M x N$ pixels, $x(m, n)$ is the intensity of the pixel in row $\mathrm{m}$ and column $\mathrm{n}$ of the image and $y(u, v)$ is the DCT coefficient in row $\mathrm{u}$ and column $\mathrm{v}$ of the DCT matrix [8].

$y(u, v)=$

$\sqrt{\frac{2}{M}} \sqrt{\frac{2}{N}} \alpha_{u} \alpha_{v} \sum_{u=0}^{M-1} \quad \sum_{v=0}^{N-1} x(m, n) \quad \cos \frac{(2 m+1) u \pi}{2 M} \cos \frac{(2 n+1) v \pi}{2 N}$

where

$$
\begin{aligned}
& \alpha_{u}=\frac{1}{\sqrt{2}} \text { if } u=0 \text { or } \alpha_{u}=1 \text { if } u=1,2, \ldots, N-1 . \\
& \alpha_{v}=\frac{1}{\sqrt{2}} \text { if } u=0 \text { or } \alpha_{v}=1 \text { if } v=1,2, \ldots, N-1 .
\end{aligned}
$$

The image is reconstructed by applying inverse DCT operation according to equation (4).

$$
\begin{aligned}
& x(m, n)= \\
& \sqrt{\frac{2}{M}} \sqrt{\frac{2}{N}} \sum_{u=0}^{M-1} \quad \sum_{v=0}^{N-1} \alpha_{u} \alpha_{v} y(u, v) \cos \frac{(2 m+1) u \pi}{2 M} \cos \frac{(2 n+1) v \pi}{2 N}
\end{aligned}
$$

The popular block-based DCT transform segments an image on non-overlapping blocks and applies DCT to each block. These results in giving three frequency sub-bands: low frequency sub-band, mid-frequency sub-band and high frequency sub-band. DCT-based watermarking is based on two facts. The first fact is that much of the signal energy lies at low-frequencies sub-band which contains the most important visual parts of the image. The second fact is that high frequency components of the image are usually removed through compression and noise attacks. The watermark is therefore embedded by modifying the coefficients of the middle frequency sub-band so that the visibility of the image will not be affected and the watermark will not be removed by compression [9]-[12].

In discrete cosine transform, the host image as shown in Fig. 6 is divided into 8 by 8 blocks and the DCT coefficients are computed for each block. The DCT value of original image as shown in Fig. 7 is done by using the DCT of each block. Discrete cosine transform can be used to reconstruct a sequence very accurately from only a few DCT coefficients. It is a useful property for applications requiring data reduction.

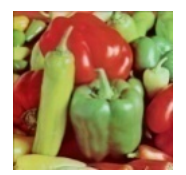

Fig. 6. Original image

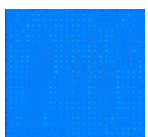

Fig. 7. DCT coefficients of original image

C. Watermark Embedding Process

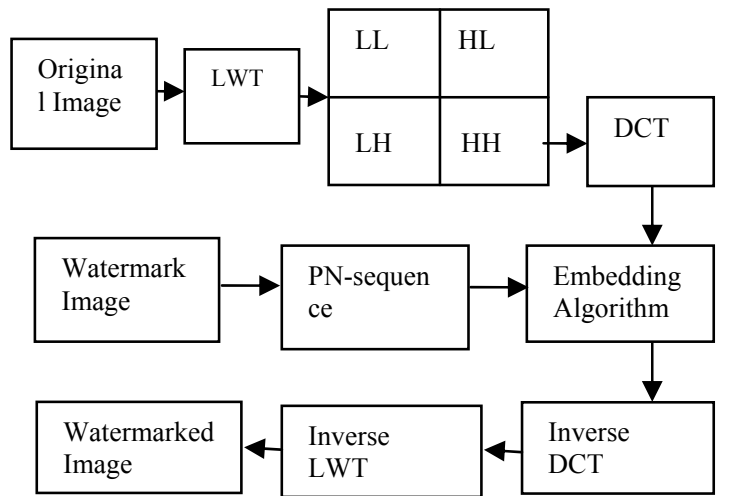

Fig. 8. Watermark embedding procedure using combined LWT-DCT method

The watermark embedding procedure is depicted in Fig. 8 followed by a detailed explanation.

Step 1: Read in the cover image $f(x, y)$.

Step 2: Apply LWT to decompose the cover image $f$ into four non-overlapping multi-resolution sub-bands: LL, HL, LH, and HH. From the four sub-bands, choose HH sub-band for embedding the watermark image. 
Step 3: Divide the sub band HH into $4 \mathrm{x} 4$ blocks.

Step 4: Apply DCT to each $4 \times 4$ block in the chosen sub-band (HH).

Step 5: Read in the binary watermark image.

Step 6: Generate two uncorrelated pseudorandom (PN) sequences. One sequence is used to embed the watermark bit 0 (PN0) and the other sequence is used to embed the watermark bit 1 (PN1). The number of elements in each of the two pseudorandom sequences must be equal to the number of mid-band elements of the DCT transformed LWT sub bands.

Step 7: Embed the two pseudorandom sequences, $P N 0$ and $P N 1$, with a gain factor, in the DCT transformed $4 \times 4$ blocks of the selected LWT sub-bands of the host image. Embedding is not applied to all coefficients of the DCT block, but only to the mid-band DCT coefficients. If $X$ is denoted as the matrix of the mid-band coefficients of the DCT transformed block, then embedding is done according to equation (5) and (6).

If the watermark bit is 0 ,

Otherwise,

$$
X^{\prime}=X+k \times P N 0
$$

$$
X^{\prime}=X+k \times P N 1
$$

where $X^{\prime}$ is watermarked DCT block.

Step 8: Apply inverse DCT (IDCT) to each block after its mid-band coefficients have been modified to embed the watermark bits as described in the previous step.

Step 9: Apply the inverse LWT (ILWT) on the LWT transformed image, including the modified sub-band, to produce the watermarked host image.

\section{Watermark Extraction Process}

The extraction of watermark image from the watermarked image is depicted in Fig. 9 and detailed in this subsection. As the proposed system is blind, it does not require the original image along with the watermarked image and the size of the watermark image for extraction.

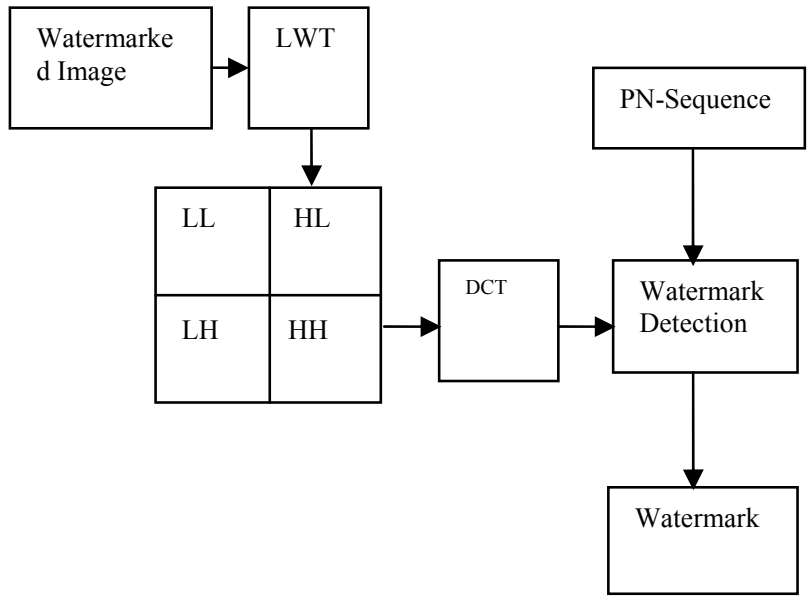

Fig. 9. Watermark extraction procedure using combined LWT-DCT method

Step 1: Apply LWT to decompose the watermarked image into four non-overlapping multi-resolution sub-bands: LL, $\mathrm{HL}, \mathrm{LH}$, and $\mathrm{HH}$.

Step 2: Divide the sub-band HH into $4 \times 4$ blocks.

Step 3: Apply DCT to each block in the chosen sub-band
$\mathrm{HH}$, and extract the mid-band coefficients of each DCT transformed block.

Step 4: Regenerate the two pseudorandom sequences $P N 0$ and PN1 using the same seed used in the watermark embedding procedure.

Step 5: For each block in the sub band HH, calculate the correlation between the mid-band coefficients and two generated pseudorandom sequences ( $P N 0$ and $P N 1)$. If the correlation with the $P N 0$ is higher than the correlation with $\mathrm{PN} 1$, then the extracted watermark bit is considered 0 , otherwise the extracted watermark is considered 1.

Step 6: Reconstruct the watermark using the extracted watermark bits and compute the similarity between the original and extracted watermark.

\section{EXPERIMENTAL RESULTS}

The experimental results of the proposed system are presented in this section.

The proposed method is tested on different images. Lena, Peppers, Opera, Kid, Flight, Bear, Rose, Pills, Water and Wildflowers are used as original images and Mandrill is applied as watermark. The proposed method makes embedding the watermark into the original image to protect owner's information and extracts it back from the watermarked image. The watermarked images of the system have a great virtue of imperceptibility, which refers to the perceived quality of the cover image in the presence of the watermark, as shown in Table I which depicts ten different original images of size $256 \times 256$, the watermark image of size $64 \times 64$, the watermarked image, and the extracted watermark.

The peak signal to noise ratio (PSNR) is typically used as a measure of the quality of a watermarked image. PSNR in decibels $(\mathrm{dB})$ is given by using equation (7) and equation (8).

$$
\begin{aligned}
& M S E=\frac{\sum[f(x, y)-F(x, y)]^{2}}{N^{2}} \\
& P S N R=20 \log _{10}\left(\frac{255}{\sqrt{M S E}}\right)
\end{aligned}
$$

The original image is $f(x, y)$ and the watermarked image is $F(x, y) . N$ is the number of pixels. MSE means Mean Square Error. The efficiency of the proposed system is illustrated by showing PSNR value. Table II depicts the names of original images with their respective PSNR values of the watermarked image obtained using proposed system.

TABLE II: PSNR VALUES FOR DIFFERENT TESTED IMAGES
\begin{tabular}{|c|c|c|}
\hline No. & Names of Images & PSNR(dB) \\
\hline 1 & Lena & 46.7629 \\
\hline 2 & Peppers & 46.3125 \\
\hline 3 & Opera & 45.8921 \\
\hline 4 & Kid & 45.7928 \\
\hline 5 & Flight & 46.2748 \\
\hline 6 & Bear & 47.3425 \\
\hline 7 & Rose & 46.7392 \\
\hline 8 & Pills & 47.4357 \\
\hline 9 & Water & 45.3241 \\
\hline 10 & Wildflowers & 45.5470 \\
\hline
\end{tabular}


TABLE I: ORIGINAL IMAGE, WATERMARK, WATERMARKED IMAGE, EXTRACTED WATERMARK, AND IMAGE AFTER EXTRACTING WATERMARK

\begin{tabular}{|c|c|c|c|c|}
\hline Original Image & Watermark & Watermarked Image & $\begin{array}{r}\text { Extracted } \\
\text { watermark }\end{array}$ & $\begin{array}{c}\text { Image after } \\
\text { extracting watermark }\end{array}$ \\
\hline Lena & Mandrill & Watermarked Lena & Extracted Mandrill & $\begin{array}{c}\text { Lena after } \\
\text { watermark retrieval }\end{array}$ \\
\hline Peppers & Mandrill & Watermarked Peppers & Extracted Mandrill & $\begin{array}{c}\text { Peppers after } \\
\text { watermark retrieval }\end{array}$ \\
\hline Opera & Mandrill & Watermarked Opera & Extracted Mandrill & $\begin{array}{c}\text { Opera after } \\
\text { watermark retrieval }\end{array}$ \\
\hline & Mandrill & Watermarked Kid & Extracted Mandrill & $\begin{array}{c}\text { Kid after watermark } \\
\text { retrieval }\end{array}$ \\
\hline & Mandrill & Watermarked Flight & $\frac{1+2}{\text { Extracted Mandrill }}$ & $\begin{array}{l}\text { Flight after } \\
\text { watermark retrieval }\end{array}$ \\
\hline Bear & Mandrill & Watermarked Bear & Extracted Mandrill & $\begin{array}{c}\text { Bear after watermark } \\
\text { retrieval }\end{array}$ \\
\hline Rose & Mandrill & Watermarked Rose & Extracted Mandrill & $\begin{array}{c}\text { Rose after } \\
\text { watermark retrieval }\end{array}$ \\
\hline 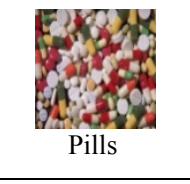 & Mandrill & $\begin{array}{c}\text { ENoy } \\
\text { Watermarked Pills }\end{array}$ & $\underbrace{}_{\text {Extracted Mandrill }}$ & $\begin{array}{c}\text { Pot } \\
\text { Pills after watermark } \\
\text { retrieval }\end{array}$ \\
\hline Water & Mandrill & Watermarked Water & Extracted Mandrill & $\begin{array}{c}\text { Water after } \\
\text { watermark retrieval }\end{array}$ \\
\hline Wildflowers & Mandrill & 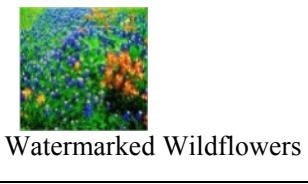 & $\begin{array}{c}\text { Extracted Mandrill } \\
\text { Ex }\end{array}$ & $\begin{array}{c}\text { Wildflowers after } \\
\text { watermark retrieval } \\
\text { W }\end{array}$ \\
\hline
\end{tabular}

\section{CONCLUSION}

In this paper, an approach for copyright protection of digital images using combined Lifting Wavelet Transform and Discrete Cosine Transform is executed. Lifting wavelet transform is used to decompose the original image. Discrete cosine transform is applied on the selected LWT sub-bands. The watermark image is embedded in the DCT transformed of the selected LWT sub-band of the original image. Subsequently, the watermark image is extracted from the watermarked image. The proposed system is blind watermarking and it does not use original image for extraction. The efficiency of the proposed system is established with the help of experimental results.

\section{REFERENCES}

[1] G. Gupta and H. Aggarwal, "Digital image watermarking using two dimensional discrete wavelet transform, discrete cosine transform and fast fourier transform," in Proc. International Journal of Recent Trends in Engineering, vol. 1, no. 1, May 2009.

[2] P. Lam, O. Winkelmeyer, S. A. Mehdi, and N. kamoosi, Watermarking technologies-analysis and design report, 2005.

[3] R. Hovancak and D. Levicky, Comparison of Watermarking Methods Using DCT Transformation, 2003. 
[4] Y. Jie, Algorithm of Image Information Hiding Based on New Anti-Arnold Transform and Blending in DCT Domain, 2009.

[5] S. Hajjara, M. Abdallah, and A. Hudaib, "Digital image watermarking using localized biorthogonal wavelets," European Journal of Scientific Reserch, vol. 26 no. 4, pp. 594-608, 2009.

[6] D. Arya, "A survey of frequency and wavelet domain digital watermarking techniques," in Proc. International Journal of Scientific and Engineering Research, vol. 1, Issue 2, November 2010.

[7] A. Phadikar, S. P. Maity, and M. K. Kundu, "Quantization based data hiding scheme for efficient quality access control of images using DWT via Lifting," in Proc. Sixth Indian Conference on Computer Vision, Graphics and Image Processing, Bhubaneswar, India, pp: 265272, 2008.

[8] P. Yip and K. Rao, Discrete Cosine Transform: Algorithms, Advantages, Applications, Academic Press, USA, 1990.

[9] W. Chu, "DCT-Based image watermarking using sub-sampling," in Proc. IEEE Trans. Multimedia, vol. 5, no. 1, pp. 34-38, 2003.

[10] S. Lin and C. Chin, "A robust DCT-based watermarking for copyright protection," in Proc. IEEE Trans. Consumer Electronics, vol. 46, no. 3, pp. 415-421, 2000.

[11] B. Wang and F. Deng, "A novel technique for robust image watermarking in the DCT domain," in Proc. IEEE 2003 Int. Conf. on Neural Networks and Signal Processing, vol. 2, pp: 1525-1528, 2003.
[12] W. Hsieh and C. Wu, "Digital watermarking using zero tree of DCT," in Proc. IEEE Trans. Consumer Electronics, vol. 46, no. 1, pp. 87-94 2000.

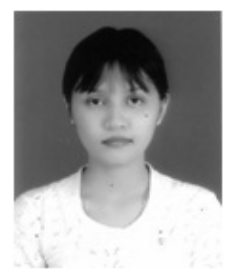

Amy Tun earned B. C. Tech (Hons) and M. C. Tech in computer technology from the University of Computer Studies, Yangon. She is a $\mathrm{PhD}$ candidate from University of Computer Studies, Yangon. She is currently working her research. Her research interests are in the areas of Digital Image Processing and Digital Image Watermarking.

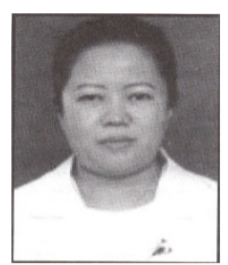

Yadana Thein is an associate professor in University of Computer Studies, Yangon under Ministry of Science and Technology, Myanmar. She is particularly interested in Digital Image Processing, Optical Character Recognition, Speech Processing, and Networking. She published about 30 papers in workshops, conferences and journals. Currently, she teaches image processing and networking subjects tounder-graduate and post-graduate students. She supervises Master theses and $\mathrm{PhD}$ researches in the areas of Digital Image Processing. 